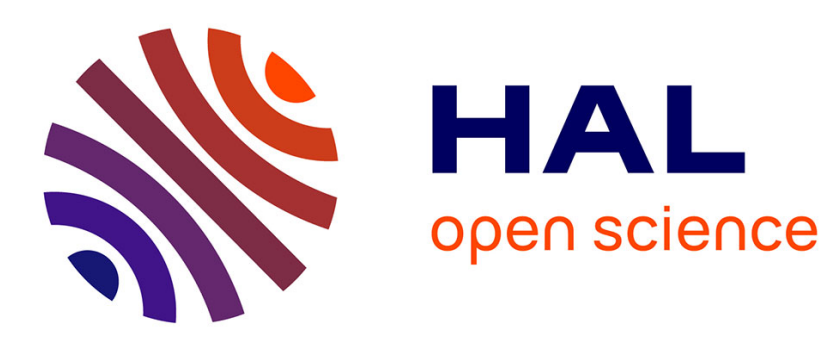

\title{
A study of a new system for measuring semiconductor using laser stimulated scattering microscope
}

\author{
T. Tanaka, A. Harata, T. Sawada
}

\section{To cite this version:}

T. Tanaka, A. Harata, T. Sawada. A study of a new system for measuring semiconductor using laser stimulated scattering microscope. Journal de Physique IV Proceedings, 1994, 04 (C7), pp.C7-171-C7174. 10.1051/jp4:1994741 . jpa-00253270

\section{HAL Id: jpa-00253270 https://hal.science/jpa-00253270}

Submitted on 1 Jan 1994

HAL is a multi-disciplinary open access archive for the deposit and dissemination of scientific research documents, whether they are published or not. The documents may come from teaching and research institutions in France or abroad, or from public or private research centers.
L'archive ouverte pluridisciplinaire HAL, est destinée au dépôt et à la diffusion de documents scientifiques de niveau recherche, publiés ou non, émanant des établissements d'enseignement et de recherche français ou étrangers, des laboratoires publics ou privés. 


\title{
A study of a new system for measuring semiconductor using laser stimulated scattering microscope
}

\author{
T. Tanaka, A. Harata and T. Sawada \\ Department of Industrial Chemistry, Faculty of Engineering, University of Tokyo, 7-3-1 Hongo, \\ Bunkyo-ku, Tokyo 113, Japan
}

\begin{abstract}
At the initial part of the transient reflecting grating signal of silicon, a signal attributed to photoexcited carrier was observed apart from thermal and acoustic effects. From theoretical approach, we found that the intensity of the carrier signal was related to the Auger recombination rate. With this signal, we obtained both images representing picosecond-photoexcited carrier concentration and Auger recombination rate of a helium-implanted crystalline silicon (dose, $10^{15}$ atoms $/ \mathrm{cm}^{2}$ ).
\end{abstract}

\section{Introduction}

We have reported that picosecond photothermal relaxation processes were observed at the initial part of transient reflecting grating (TRG) signals [1]. TRG method, like a transient grating method, is a powerful tool to observe ultra-fast transport phenomena [1-3]. It has an advantage over TG of surface (interface) selectivity [4], because of using reflecting diffraction. TRG also has a merit in high spatial resolution as small as laser spot size, hence we can use TRG for surface selective microscopic measurements [5]. We have called this method laser-stimulated scattering microscope (LSSM). Images of ionimplanted silicon by LSSM have previously reported using thermal effect of the TRG signal [5,6]. For silicon, we recently detected a signal attributed to photoexcited carrier apart from those of thermal and acoustic effects [1]. In this report, we treat the carrier signal theoretically, and relate the intensity with Auger recombination (AR) rate of the sample surface. We also find that helium-ion implantation into silicon increases the AR rate, while thermal signal changes a little. Additionally, with this carrier signal, we first obtain both images representing photoexcited carrier concentration and AR rate .

\section{Experimental}

A block diagram of LSSM is shown in Fig. 1. A frequency-doubled mode-locked and Q-switched $\mathrm{Nd}$ :YAG laser was used for our experiment. Light pulses (wavelength $\lambda, 532 \mathrm{~nm}$; duration, $84 \mathrm{ps}$ ) were generated with a repetition rate of $1.03 \mathrm{kHz}$. A single pulse was divided into three parts to yield two pump pulses and a weak probe pulse. The pump pulses were $60 \mu \mathrm{m}$ in diameter and less than $300 \mathrm{~nJ} / \mathrm{pulse}$ in total energy at the maximum intensity. The energy density is low enough to prevent samples from damages. A variable neutral density (ND) filter was utilized to adjust the excitation energy. The two pump pulses were temporally and spatially overlapped at a sample surface with a crossing angle of $2 \theta$ to generate interference pattern having a fringe spacing of $\Lambda=\lambda / 2 \sin \theta$. Hence the surface was excited spatially periodically. Such a pattern cause various changes at the sample surface, i.e., excited state population, temperature rise and thermal stress inducing a counter propagating surface acoustic wave (SAW). Since each of them works as a reflecting grating, one can observe them by using the diffraction of the third laser pulse (probe) incident at the excited region. The diffracted light was detected to obtain a transient response with 
scanning a computer-controlled optical delay line. For mapping, we fixed the optical delay line at an adequate position and scanned the sample stage. Spatial resolution depends on the spot size of probe pulse, and was $40 \mu \mathrm{m}$ at this time. The samples measured were a silicon single crystals (111) undergoing 200 $\mathrm{keV}$ argon- or helium-ion implantation at room temperature with a dose of $10^{15}$ and $10^{13}$ atoms $/ \mathrm{cm}^{2}$, respectively. The implanted region was restricted by a $6 \mathrm{~mm} \phi$ holed aluminum mask. No further treatments were performed after the implantation.

\section{Results and discussion}

\subsection{Laser power dependence of TRG} signal

In Fig. 2, we show typical TRG signal of the intrinsic (a) and the implanted region (b) of the helium-ion implanted silicon (He-Si). The peak, indicated by $\mathrm{X}$ and observed at the time nearly zero, was attributed to a concentration grating of photoexcited carrier. The signal after the peak indicated by $Y$ consists of two components: decaying and oscillating. The decaying components is a signal from thermal grating, and its lifetime is related to both thermal diffusivity of sample and the fringe spacing of TRG. The oscillating component is due to counter propagating SAW in $\mathrm{GHz}$ order. We have been able to separate these parts from a TRG signal. Since the intensity of signal $\mathrm{Y}$ increases more than hundreds times when argon ions are implanted into silicon (111) $\left(10^{15}\right.$ atoms $\left./ \mathrm{cm}^{2}\right)$, it is very useful to use the signal $Y$ to evaluate ion implantation [7]. On the other hand, the intensity of signal $X$ drastically decreases by helium ion implantation to silicon (111) (dose of $10^{15}$ atoms $/ \mathrm{cm}^{2}$ ) even when the change of the signal $Y$ is a little. In short, the signal $X$ is more sensitive than signal $Y$ to ion implantation.

We measure both excitation and probe power dependence of TRG signal intensity using ND filters. From a simple consideration, intensity of diffracted signal (S) from transient gratings is proportional to the probe pulse intensity (Ip) and square of excitation pulse intensity (Ie), i.e., $S \propto$ Ie $\times$ Ie $\times$ Ip. It was

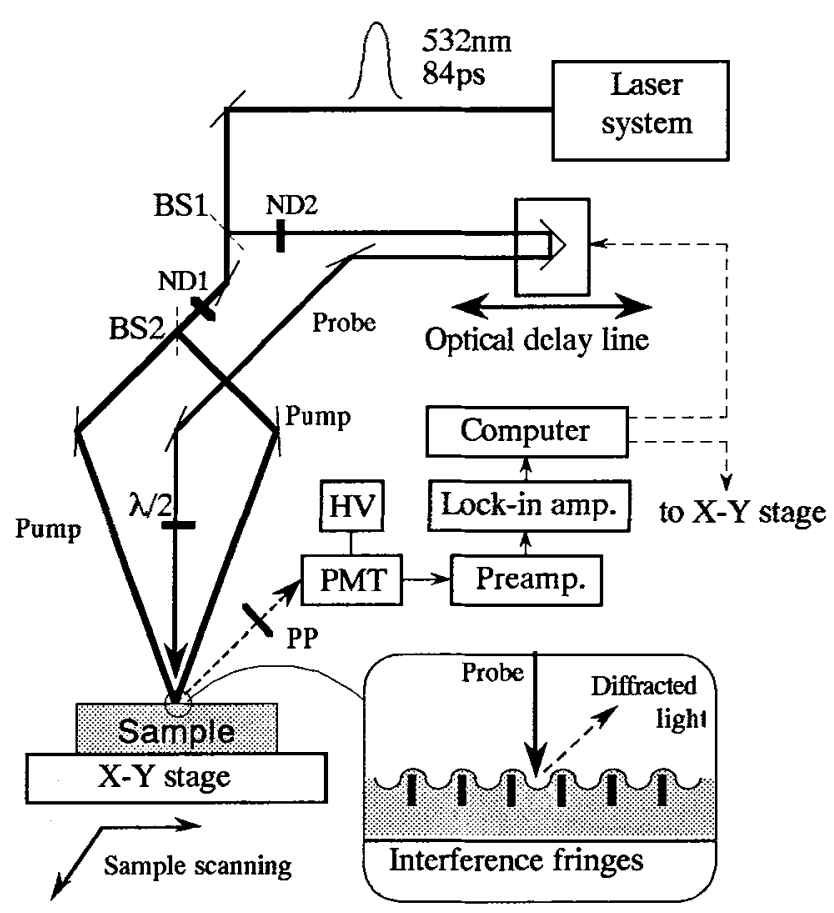

Fig. 1. Experimental setup for LSSM. The laser system consists of a Q-switched and mode-locked Nd:YAG laser, a frequency doubler and a single pulse selecter. BS, beam splitter; ND, neutral density filter; PMT, photomultiplier; HV, high voltage supply; PP, polarizer and $\lambda / 2$, half wave plate. The other optical elements are prisms or mirrors. The inset shows the arrangements of the grating and probe light.

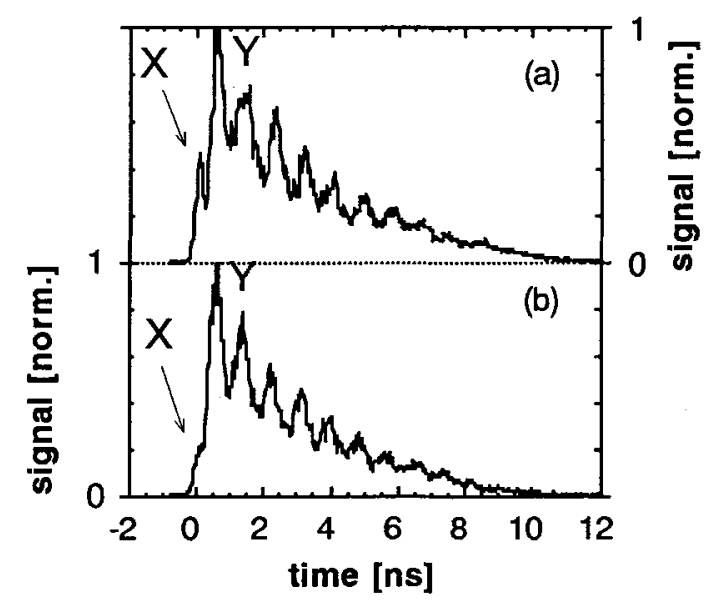

Fig. 2. Typical TRG signal for thehelium ion-implanted silicon, non-implanted (a) and implanted region (b). 
experimentally confirmed that the intensity of both signal $X$ and $Y$ were proportional to Ip. Figure 3 shows excitation power dependence of signal $\mathrm{X}$ intensity for the argon ion-implanted silicon. Solid lines represent theoretical curves. Signal $\mathrm{X}$ intensity is proportional to square of excitation pulse power up to $5 \mathrm{~mJ} / \mathrm{cm}^{2}$, and a saturation is observed beyond this point. We assume that the saturation of the carrier signal was caused by a nonlinear process in carrier recombination.

\subsection{Theoretical}

In this section we will set up equations for carrier density and temperature at silicon surface. With a supposition that each of incident photons create a carrier, the photoexcited carrier concentration will be up to $10^{20} / \mathrm{cm}^{3}$ at the maximum energy

density. At this concentration, a rate equation of carriers has to include AR process. We make the equation under three assumption that one absorbed-photon makes a carrier, excess photon energy above the energy gap convert to thermal energy in a moment via phonons, and that carrier recombination is non-radiative. Surface recombination is estimated to be slow enough to be ignore comparing with AR process. We only take into account of diffusion perpendicular to gratings, and neglect those parallel to grating and into depth direction. The equations of carrier density and temperature including the one-dimensional diffusion are as follows:

$$
\begin{aligned}
& \frac{\partial N}{\partial t}=-\gamma_{1} N-\gamma_{2} N^{2}-\gamma_{3} N^{3}+D \frac{\partial^{2} N}{\partial x^{2}}+(1-R) I(t) \\
& \rho C \frac{\partial T}{\partial t}=-E_{g} \frac{\partial N}{\partial t}+\kappa \frac{\partial^{2} T}{\partial x^{2}}+\left(h v-E_{g}\right)(1-R) I(t)
\end{aligned}
$$

where $N$ is carrier density, $D$ is carrier diffusivity, $\gamma_{1}, \gamma_{2}$ and $\gamma_{3}$ are carrier recombination rate of linear, two-body and Auger processes, respectively, $T$ is temperature, $E_{g}$ is energy gap for silicon, $R$ is optical reflectivity, $\rho$ is density, $C$ is heat capacity, $\kappa$ is thermal conductivity, $h v$ is photon energy and $I(t)$ is time profile of incident photon number. The axis $x$ is parallel to surface and perpendicular to interference fringes. Nonlinear couplings between $T$ and $N$ are ignored.

We calculate these equations with all parameters in literature data $[8,9]$ by a personal computer. These equations are numerically integrate by time to get the time profiles of $N$ and $T$. The results were compared with the experimental data. The calculated time profile of carrier density is in good agreement with experimental data of signal $X$. We also calculated maximum carrier density $\left(\mathrm{N}_{\max }\right)$ as a function of incident laser power, and fit the calculated data to the experimental such. Since the AR is a dominant process at such a high carrier concentration, $\gamma_{3}$ is only decisive parameter in Eqs. The estimated $\gamma_{3}$ is $4 \times 10^{-29}$ $\mathrm{cm}^{6} / \mathrm{s}$ for intrinsic silicon. The obtained $\gamma_{3}$ is two orders of magnitude higher than other reported experimental or calculated values which was around $4 \times 10^{-31} \mathrm{~cm}^{6} / \mathrm{s}[10]$. We are considering that defects generated by surface polishing act as a recombination center and accelerate the AR process. There might be the other surface effects such as an effect of surface oxide. The solid line in Fig. 3 is the fitted data for argon ion-implanted silicon, and obtained $\gamma_{3}$ was $7 \times 10^{-29} \mathrm{~cm}^{6} / \mathrm{s}$. This value is about two times larger than intrinsic. AR rate was increased with defects induced by ion implantation. We conclude that signal $\mathrm{X}$ is a signal from photoexcited carrier which decreases with increasing $\gamma_{3}$, and that ion implantation increase AR rate via the generation of defects near the surface. 


\subsection{Imaging}

We have shown that signal $\mathrm{X}$ is due to photoexcited carrier. Using the signal $\mathrm{X}$, the images of photoexcited carrier is available by LSSM measurements. Figure 4 shows an LSSM image of signal X for helium-ion-implanted silicon (He-Si), scanning area is $10 \times 10\left[\mathrm{~mm}^{2}\right]$ by $100 \times 100$ pixcels, and (b) illustrates the implanted region. Figure 4 (a) is the image of picosecond photoexcited carrier, obtained for the first time. We had been reported that thermal signal is very sensitive to defects made by ion implantation; we can detect the change of signal $\mathrm{Y}$ by $300 \mathrm{keV}$ argon-ion implantation at a dose as low as $10^{11}$ atoms $/ \mathrm{cm}^{2}$ [7]. In comparing two TRG signals in Fig. 2, although increase of signal Y intensity is small and SAW frequency change is only detection limit, decrease of signal $\mathrm{X}$ intensity is obvious. Thus contrast of signal X image is clearer than $Y^{\prime}$ 's. Here we emphasize that the signal from photoexcited carrier is more sensitive to defects than the thermal. Furthermore we can easily obtain a calibration curve between $N_{\max }$ and $\gamma_{3}$ by calculating Eq. 1 . From this curve, the image of carrier concentration is converted to the image of $\gamma_{3}$. Thus we can obtain an image of AR rate.

(a)

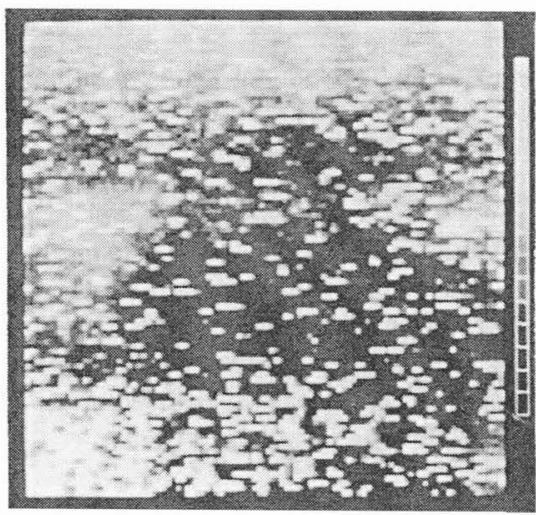

(b)

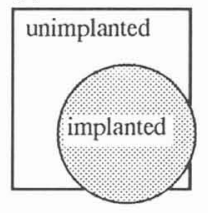

Fig. 4. Image of helium ion implanted silicon (He-Si) by signal X (a) . Figure (b) scematically shows the implanted region. Scanning area is $10 \times 10\left[\mathrm{~mm}^{2}\right]$ by $100 \times 100$ pixcels.

\section{Conclusions}

We have separately detected the signal attributed to photoexcited carricr apart from the thermal and acoustic effects by picosecond time-resolved TRG measurement. Theoretical calculation shows that the peak at nearly time zero is the signal due to photoexcited carriers, and that its intensity decrease with increasing Auger recombination rate, $\gamma_{3}$. We can estimate $\gamma_{3}$ by measuring the relation between the intensity of signal $X$ and the excitation energy. At the surface of silicon there must be some defects which increase $\gamma_{3}$. Helium ion implantation also increase $\gamma_{3}$, corresponding to defects increase, on silicon surface. Using this signal $\mathrm{X}$ for imaging, we obtain an image of picosecond photoexcited carrier. The image can be easily converted to the image of $A R$ rate.

\section{References}

[1] Nishimura H., Harata A. and Sawada T., Jpn. J. Appl. Phys. 32 (1993) 5149-5154

[2] Eichler H. J,.Günter P. and Pohl D. W., Laser-Induced Dynamic Gratings (Springer-Verlag, Berlin, 1986)

[3] Harata A. and Sawada T., Appl. Phys. Lett. 58 (1991) 1839-1841

[4] Fishman I. M., Marshall C. D., Meth J. S. and Fayer M. D., J. Opt. Soc. Am. B 8 (1991) 18801888

[5] Harata A., Nishimura H., Tanaka T. and Sawada T., Rev. Sci. Instrum. 64 (1993) 618-622

[6] Harata A., Shen Q., Tanaka T. and Sawada T., Jpn. J. Appl. Phys. 32(1993) 3633-3638

[7] Nishimura H., Harata A. and Sawada T., Jpn. J. Appl. Phys. 31, Suppl. 31-1 (1992) 91-93

[8] Hellwege K. H., Landort Börnstain New Series-17 (Springer-Verlag Berlin, 1982) pp. 43-87

[9] Woerdman J. P., Some Optical and Electrical Properties of a Laser-Generated Free-Carrier Plasma in Si (Philips Res. Rep's Suppl. No. 7, 1971) pp. 12-16

[10] Lakes D. B., Neumark G.F.and Pantelides S. T., Phys. Rev. B. 42 (1990) 5176-5185 